\title{
Performance Analysis on Output SINR of Robust Two-Stage Beamforming
}

\author{
Tsui-Tsai Lin ${ }^{1}$, Fuh-Hsin Hwang ${ }^{2}$, Juinn-Horng Deng ${ }^{3}$ \\ ${ }^{1}$ Department of Electronic Engineering, National United University, Miaoli, Taiwan; ${ }^{2}$ Department of Optoelectronics and Communi- \\ cation Engineering, National Kaohsiung Normal University, Kaohsiung, Taiwan; ${ }^{3}$ Department of Communication Engineering, Yuan \\ Ze University, Taoyuan, Taiwan. \\ Email: ttlincs@nuu.edu.tw
}

Received October $30^{\text {th }}, 2010$; revised January $3^{\text {rd }}, 2011$; accepted January $4^{\text {th }}, 2011$

\begin{abstract}
In this paper, we present a theoretical analysis of the output signal-to-interference-plus-noise ratio (SINR) for eigenspace beamformers so as to investigate the performance degradation caused by large pointing errors. For the sake of reducing such performance loss, a robust scheme, which consists of two cascaded signal processors, is proposed for adaptive beamformers. In the first stage, an algorithm possessing time efficiency is developed to adjust the direction-of-arrival (DOA) estimate of the desired source. Based the achieved DOA estimate, the second stage provides an eigenspace beamformer combined with the spatial derivative constraints (SDC) to further mitigate the cancellation of the desired signal. Analysis and numerical results have been conducted to verify that the proposed scheme yields a better robustness against pointing errors than the conventional approaches.
\end{abstract}

Keywords: Beamforming, Large Pointing Error, Output Signal-To-Interference-Plus-Noise Ratio (SINR), Eigenspace Beamformer, Two-Stage

\section{Introduction}

It is well known that conventional adaptive beamfomers are effective in suppressing strong interferers as long as the error in the steering vector due to pointing inaccuracy is small [1]. In the presence of steering vector errors, these beamformers exhibit severe degradation in performance in that the output signal-to-interference-plusnoise ratio (SINR) drops dramatically. Remedies have been proposed to lessen the effect of desired signal cancellation [2]. In particular, the linearly constrained minimum variance (LCMV) beamformer uses the spatial derivative constraints (SDC) to alleviate sensitivity to the pointing errors by means of a flatter main-lobe response $[3,4]$. Unfortunately, this in turn results in large sidelobes and leads to a loss in array gain against noise. Furthermore, the decrement in the beamwidth as the input signal-to-noise ratio (SNR) increases makes it poor in interference suppression due to the directional mismatch [5]. In the extreme case of high input SNR, since the desired signal may fall outside the main-lobe region, the SDC beamformer would null out not only the interference but also the desired signal. In [6], a robust scheme employs the leaky elimination constraint and the interference null constraint to preserve the desired signal and to keep nice interference nulling simultaneously. This method needs to identify the interference subspace for the sake of restoring the interference correlation matrix. However, in the presence of weak interference, it is difficult to extract the interference subspace from the received data. Chang and Yeh [7] proposed the eigenspace beamformer, in which the weight vector is constrained within the signal subspace of the received data correlation matrix. In spite of success in dealing with a moderate pointing error, this approach cannot completely remove the interference and the residual interference impairs the system performance, especially for the weak interference (low signal-to-interference ratio, SIR). To mitigate the effect of large point errors, an iterative searching method [8] is considered for constructing the correct constraint vector before beamforming. Its low convergence behavior because of a large pointing error becomes crucial in practice. This approach, at the worst, breaks down when the desired signal falls outside the main-beam region in the case of high input SNR and/or a large antenna array.

In this paper, the effect of pointing errors on the eigenspace beamformer is first investigated by using the theoretical analysis. As a remedy, a robust two-stage 
scheme for adaptive beamformer is proposed [9]. The design of this beamformer involves the following procedure. First, an accuracy direction-of-arrival (DOA) estimate is determined from a few angles-of-interest in accordance with the fact that the output power of the beamformer decreases with the increment in the pointing error. By exploiting the refined resultant, an eigenspace beamformer incorporating with the SDC is used to further mitigate the aggregate impacts due to the pointing errors. A closed-form approximate SINR expression is given to indicate the achievable performance improvement. Numerical results then confirm the efficacy of the proposed robust method and corroborate the predicted SINR results.

The remainder of the paper is organized as follows: Section 2 reviews the array data model and presents the SINR performance analysis of the eigenspace beamformer. The proposed two-stage beamformer and its performance analysis are provided in Section 3. Simulation results and conclusion remarks are given in Sections 4 and 5 , respectively.

The notations used in this paper follow the usually conventional-bold capital letters denoting vectors and matrices. I is an identity matrix with a proper dimension, $\operatorname{diag}\{\mathbf{v}\}$ is a diagonal matrix with its entries formed by $\mathbf{v},(\cdot)^{T}$ and $(\cdot)^{H}$ are transpose and complex conjugate transpose of $(\cdot)$. Also, $\operatorname{Re}\{\cdot\}, E\{\cdot\}$, and $\mid \cdot$ are used to denote real part, ensemble average, and absolute operators, respectively.

\section{Preliminary}

The scenario considered herein involves a single desired source and $K-1$ uncorrelated interfering sources, all assumed to be narrowband with the same center frequency. These sources are in the far field of a uniform linear array consisting of $M$ identical elements spaced by half a wavelength. Adopting the complex envelope notation, the array data obtained at a certain sampling instant can be put in the $M \times 1$ vector form:

$$
\mathbf{x}(n)=\sum_{k=1}^{K} s_{k}(n) \mathbf{a}\left(\theta_{k}\right)+\mathbf{n}(n)
$$

The random scalars $s_{k}(n)$ for $k=1,2, \cdots, K$ represent the signals with power $\sigma_{k}^{2}$. The $M \times 1$ vector $\mathbf{a}(\theta)=\left[1, e^{j \pi \sin \theta}, \cdots, e^{j(M-1) \pi \sin \theta}\right]^{T} / \sqrt{M}$ is the array steering vector, in which $\theta$ is the physical angle measured with respect to the broadside of the array. Finally, the vector $\mathbf{n}(n)$ is additive white Gaussian noise with power $\sigma_{n}^{2} \mathbf{I}$. Without loss of generality, suppose $s_{1}(n)$ is the desired signal.

The LCMV beamformer, which minimizes the array output power subject to a unit constraint on the presumed vector, is widely used to preserve the desired signal and keep interference nulling simultaneously. Mathematically speaking, the optimal weight can be obtained by [3]:

$$
\mathbf{w}=\mathbf{R}^{-1} \mathbf{a}\left(\theta_{s}\right) / \mathbf{a}^{H}\left(\theta_{s}\right) \mathbf{R}^{-1} \mathbf{a}\left(\theta_{s}\right),
$$

where $\mathbf{a}\left(\theta_{s}\right)$ is the steering vector associated with the look direction $\theta_{s}$, and $\mathbf{R}$ is an $M \times M$ received data correlation matrix given by

$$
\mathbf{R}=E\left\{\mathbf{x}(n) \mathbf{x}^{H}(n)\right\}=\sum_{k=1}^{K} \sigma_{k}^{2} \mathbf{a}\left(\theta_{k}\right) \mathbf{a}^{H}\left(\theta_{k}\right)+\sigma_{n}^{2} \mathbf{I} .
$$

According to the orthogonality between the signal and noise subspaces, the eigenspace technique can be used to mitigate the effect of desired signal cancellation and its corresponding weight vector is given by

$$
\begin{aligned}
\mathbf{w}_{s} & =\mathbf{E}_{s} \mathbf{E}_{s}^{H} \mathbf{w} \\
& =\mathbf{E}_{s} \mathbf{E}_{s}^{H} \mathbf{R}^{-1} \mathbf{a}\left(\theta_{s}\right) / \mathbf{a}^{H}\left(\theta_{s}\right) \mathbf{R}^{-1} \mathbf{a}\left(\theta_{s}\right),
\end{aligned}
$$

where the $M \times K$ matrix $\mathbf{E}_{s}$ is formed by the $K$ principal eigenvectors of $\mathbf{R}$. Under proper conditions, the eigenspace beamformer is found to achieve high output SINR as long as the pointing error $\left(\theta_{s}-\theta_{1}\right)$ is negligible. Unfortunately, in the case of the large pointing error and/or high input SNR, its performance is limited mostly by the residual interference buried in the beamformer output.

To gain further insights, we will describe the effect of the residual interference caused by pointing inaccuracy. For a manageable analysis, the scenario is simplified into that involving a desired source with power $\sigma_{1}^{2}=1$ and an interferer with power $\sigma_{2}^{2}$ only, i.e., $K=2$. Thus the received data correlation matrix can be rewritten as

$$
\mathbf{R}=\mathbf{a}\left(\theta_{1}\right) \mathbf{a}^{H}\left(\theta_{1}\right)+\sigma_{2}^{2} \mathbf{a}\left(\theta_{2}\right) \mathbf{a}^{H}\left(\theta_{2}\right)+\sigma_{n}^{2} \mathbf{I} .
$$

In addition, for the ease of expression, the following notations are defined: $\mathbf{a}_{k}=\mathbf{a}\left(\theta_{k}\right)$ and $\mathbf{a}_{k}^{H} \mathbf{a}_{j}=\rho_{k j}$ for $k, j=1,2, s$. Note that $\rho_{k j}$ denotes the correlation between $\mathbf{a}_{k}$ and $\mathbf{a}_{j}$, and is close to zero for a well-separated sources.

By using some algebraic manipulations, the received data correlation matrix can be decomposed as

$$
\begin{aligned}
\mathbf{R} & =\lambda_{1} \mathbf{e}_{1} \mathbf{e}_{1}^{H} / \mathbf{e}_{1}^{H} \mathbf{e}_{1}+\lambda_{2} \mathbf{e}_{2} \mathbf{e}_{2}^{H} / \mathbf{e}_{2}^{H} \mathbf{e}_{2}+\sigma_{n}^{2} \mathbf{I} \\
& =\lambda_{1} \mu_{1} \mathbf{e}_{1} \mathbf{e}_{1}^{H}+\lambda_{2} \mu_{2} \mathbf{e}_{2} \mathbf{e}_{2}^{H}+\sigma_{n}^{2} \mathbf{I},
\end{aligned}
$$

where

$$
\begin{aligned}
& \lambda_{k}=\left[1+\sigma_{2}^{2}-(-1)^{k} \sqrt{\left(\sigma_{2}^{2}-1\right)^{2}+4 \sigma_{2}^{2}\left|\rho_{12}\right|^{2}}\right] / 2 ; \\
& \mathbf{e}_{k}=\mathbf{a}_{1}+\left(\lambda_{1}-1\right) \mathbf{a}_{2} / \rho_{12} ; \quad \mu_{k}=1 / \mathbf{e}_{k}^{H} \mathbf{e}_{k}, k=1,2 .
\end{aligned}
$$

Under the simplified scenario, the weight vector for the eigenspace beamformer is given by 


$$
\begin{aligned}
\mathbf{w}_{s} & =\frac{\mu_{1} \mathbf{e}_{1}^{H} \mathbf{a}_{s}}{\lambda_{1}+\sigma_{n}^{2}} \mathbf{e}_{1}+\frac{\mu_{2} \mathbf{e}_{2}^{H} \mathbf{a}_{s}}{\lambda_{2}+\sigma_{n}^{2}} \mathbf{e}_{2} \\
& =\frac{g_{1} \mu_{1}}{\lambda_{1}+\sigma_{n}^{2}} \mathbf{e}_{1}+\frac{g_{2} \mu_{2}}{\lambda_{2}+\sigma_{n}^{2}} \mathbf{e}_{2},
\end{aligned}
$$

where

$$
g_{k}=\mathbf{e}_{k}^{H} \mathbf{a}_{s}=\rho_{1 s}+\rho_{2 s}\left(\lambda_{k}-1\right) / \rho_{21}, \quad k=1,2 .
$$

Note that we have omitted the normalized scalar since it does not affect the analysis result. Using (8), the output desired signal power $P_{d}$ and the output interferenceplus-noise power $P_{\text {in }}$ are given by

$$
\begin{aligned}
P_{d} & =\mathbf{w}_{s}^{H} \mathbf{a}_{1} \mathbf{a}_{1}^{H} \mathbf{w}_{s} \\
& =\left|\frac{g_{1} \mu_{1}}{\lambda_{1}+\sigma_{n}^{2}} \mathbf{a}_{1}^{H} \mathbf{e}_{1}+\frac{g_{2} \mu_{2}}{\lambda_{2}+\sigma_{n}^{2}} \mathbf{a}_{1}^{H} \mathbf{e}_{2}\right|^{2} \\
& =\left|\frac{g_{1} \lambda_{1} \mu_{1}}{\lambda_{1}+\sigma_{n}^{2}}+\frac{g_{2} \lambda_{2} \mu_{2}}{\lambda_{2}+\sigma_{n}^{2}}\right|^{2} ; \\
P_{\text {in }} & =\mathbf{w}_{s}^{H} \mathbf{R} \mathbf{w}_{s}-P_{d} \\
& =\frac{\mu_{1}\left|g_{1}\right|^{2}}{\lambda_{1}+\sigma_{n}^{2}}+\frac{\mu_{2}\left|g_{2}\right|^{2}}{\lambda_{2}+\sigma_{n}^{2}}-\left|\frac{g_{1} \lambda_{1} \mu_{1}}{\lambda_{1}+\sigma_{n}^{2}}+\frac{g_{2} \lambda_{2} \mu_{2}}{\lambda_{2}+\sigma_{n}^{2}}\right|^{2},
\end{aligned}
$$

in which we have used the facts that $\mathbf{a}_{1}^{H} \mathbf{e}_{k}=\lambda_{k}$ for $k=1,2$. Taking the ratio of $P_{d}$ and $P_{i n}$ with substitution of (10) yields the output SINR expression:

$$
\begin{aligned}
\operatorname{SINR}_{o}= & \frac{P_{d}}{P_{i n}}=\frac{\mathbf{w}_{s}^{H} \mathbf{a}_{1} \mathbf{a}_{1}^{H} \mathbf{w}_{s}}{\mathbf{w}_{s}^{H} \mathbf{R} \mathbf{w}_{s}-P_{d}} \\
= & \frac{\left|\frac{g_{1} \lambda_{1} \mu_{1}}{\lambda_{1}+\sigma_{n}^{2}}+\frac{g_{2} \lambda_{2} \mu_{2}}{\lambda_{2}+\sigma_{n}^{2}}\right|^{2}}{\frac{\mu_{1}\left|g_{1}\right|^{2}}{\lambda_{1}+\sigma_{n}^{2}}+\frac{\mu_{2}\left|g_{2}\right|^{2}}{\lambda_{2}+\sigma_{n}^{2}}-\left|\frac{g_{1} \lambda_{1} \mu_{1}}{\lambda_{1}+\sigma_{n}^{2}}+\frac{g_{2} \lambda_{2} \mu_{2}}{\lambda_{2}+\sigma_{n}^{2}}\right|^{2}} .
\end{aligned}
$$

This result reveals that the output SINR is dependent upon the look direction $\theta_{s}\left(g_{1}\right)$, which decreases as the pointing error increases.

Under the condition that the interference is far away from the desired source, i.e, $\rho_{12} \ll 1$, we have

$$
\begin{aligned}
& \lambda_{1} \approx 1 ; \quad \lambda_{2} \approx \sigma_{2}^{2} ; \\
& \mathbf{e}_{1} \approx \mathbf{a}_{1} ; \quad \mathbf{e}_{2} \approx\left(\sigma_{2}^{2}-1\right) \mathbf{a}_{2} / \rho_{12} ; \\
& g_{1} \approx \rho_{1 s} ; \quad g_{2} \approx \rho_{2 s}\left(\sigma_{2}^{2}-1\right) / \rho_{21} .
\end{aligned}
$$

Substituting (12) into (11), the output SINR can be reduced to

$$
\operatorname{SINR}_{o} \approx \frac{\left|\frac{\rho_{1 s}}{1+\sigma_{n}^{2}}+\frac{\sigma_{2}^{2} \rho_{12} \rho_{2 s}}{\left(\sigma_{2}^{2}+\sigma_{n}^{2}\right)\left(\sigma_{2}^{2}-1\right)}\right|^{2}}{\frac{\left|\rho_{1 s}\right|^{2}}{1+\sigma_{n}^{2}}+\frac{\left|\rho_{2 s}\right|^{2}}{\sigma_{2}^{2}+\sigma_{n}^{2}}-\left|\frac{\rho_{1 s}}{1+\sigma_{n}^{2}}+\frac{\sigma_{2}^{2} \rho_{12} \rho_{2 s}}{\left(\sigma_{2}^{2}+\sigma_{n}^{2}\right)\left(\sigma_{2}^{2}-1\right)}\right|^{2}}
$$

$$
\begin{gathered}
\approx \frac{\frac{\left|\rho_{1 s}\right|^{2}}{\left(1+\sigma_{n}^{2}\right)^{2}}}{\frac{\left|\rho_{1 s}\right|^{2}}{1+\sigma_{n}^{2}}+\frac{\left|\rho_{2 s}\right|^{2}}{\sigma_{2}^{2}+\sigma_{n}^{2}}-\frac{\left|\rho_{1 s}\right|^{2}}{\left(1+\sigma_{n}^{2}\right)^{2}}} \\
=\mathrm{SNR}_{i} \times \frac{\left|\rho_{1 s}\right|^{2}\left(1+\mathrm{INR}_{i}\right)}{\left|\rho_{1 s}\right|^{2}\left(1+\mathrm{INR}_{i}\right)+\left|\rho_{2 s}\right|^{2}\left(1+\mathrm{SNR}_{i}\right)}
\end{gathered}
$$

$$
\approx\left\{\begin{array}{cc}
\mathrm{SNR}_{i}, & \mathbf{a}_{s} \approx \mathbf{a}_{1} \\
\mathrm{SNR}_{i}, & \mathrm{INR}_{i} \gg 1 \\
\frac{\sigma_{2}^{2}}{\sigma_{1}^{2}} \times \frac{\left|\rho_{1 s}\right|^{2}}{\left|\rho_{2 s}\right|^{2}} & \rho_{1 s} \ll 1 \& \mathrm{SNR}_{i} \gg 1
\end{array},\right.
$$

where $\operatorname{SNR}_{i}=1 / \sigma_{n}^{2}$ and $\operatorname{INR}_{i}=\sigma_{2}^{2} / \sigma_{n}^{2}$ denote the input SNR and interference-to-noise ratio (INR), respectively. The results in (13) reveal several intrinsic features of the eigenspace beamformer. First of all, as long as the look direction is close to the DOA of the desired source ( $\mathbf{a}_{s} \approx \mathbf{a}_{1}$ ), the eigenspace beamformer performs like the optimal quiescent beamformer, which can offer the maximum output SINR equal to $\mathrm{SNR}_{i}$. The second one is that the eigenspace beamformer can achieve a reliable performance without severe desired signal cancellation for $\mathrm{INR}_{i} \gg 1$. On the contrary, in presence of weak interference, the beamformer fails to completely remove the interference and the residual interference cannot be negligible when compared with the output noise power, leading to a substantial degradation in output SINR. Finally, in the case of high input SNR $\left(\mathrm{SNR}_{i} \gg 1\right)$ and/or large pointing errors $\left(\rho_{1 s} \ll 1\right)$, the second term of the denominator becomes large and cannot be negligible when compared with the first term. This significantly drops the performance of the eigenspace beamformer. To make matters worse, the eigenspace beamformer reaches a "saturation region", in which the output SINR is independent upon the input SNR.

\section{Proposed Robust Two-Stage Beamforming}

As mentioned above, the eigenspace beamformer cannot offer a reliable SINR performance as the error in DOA estimate is large and/or the input SNR is high, especially for weak interference. An alternative to enhancing robustness is to adjust the DOA estimate before beamforming. This prompts us to propose a two-stage scheme. In the first stage, we determine an accuracy DOA estimate based on the fact that the output power of the LCMV beamformer decreases with the increment in the pointing error. In the second stage, to mitigate desired signal cancellation, we further leverage the spatial derivative technique to incorporate the refined DOA esti- 
mate into the eigenspace beamformer.

\subsection{Proposed Two-Stage Beamformer}

According to (2), the output power of the LCMV beamformer is given by

$$
P_{o}\left(\theta_{s}\right) \approx\left[\mathbf{a}^{H}\left(\theta_{s}\right) \mathbf{R}^{-1} \mathbf{a}\left(\theta_{s}\right)\right]^{-1},
$$

which achieves a maximum output SINR when the steering vector $\mathbf{a}\left(\theta_{s}\right)$ coincides with that of the desired signal $\mathbf{a}\left(\theta_{1}\right)$ [8]. This suggests that an accuracy DOA estimate can be chosen from the candidate angle set, $S_{\theta}=\left\{\theta_{m}: \theta_{s}+m B / 2 N\right\}$ for $m=-N,-N+1, \cdots, N$ in accordance with maximizing the array output power:

$$
\begin{aligned}
\hat{\theta}_{s} & \approx \max _{\theta_{m} \in S_{\theta}}\left[\mathbf{a}^{H}\left(\theta_{m}\right) \mathbf{R}^{-1} \mathbf{a}\left(\theta_{m}\right)\right]^{-1} \\
& \equiv \min _{\theta_{m} \in S_{\theta}} \mathbf{a}^{H}\left(\theta_{m}\right) \mathbf{R}^{-1} \mathbf{a}\left(\theta_{m}\right),
\end{aligned}
$$

where $B$ denotes the presumed angle region of interesting. The choosing of $N$ is a trade-off between accuracy in DOA estimate and computational load. A small value of $N$ leads to time-saving in searching $\hat{\theta}_{s}$, but poor performance in beamforming, and vice versa. Since the major consideration in the first stage is to get rid of desired signal cancellation due to a large pointing error, a small value of $N$ is preferred.

In order to further improve robustness against the error in DOA estimation, an eigenspace beamformer is to incorporate a first-order SDC in the direction $\hat{\theta}_{s}$. We have the weight vector given by

$$
\begin{aligned}
\mathbf{w}= & \mathbf{E}_{s} \mathbf{E}_{s}^{H} \mathbf{R}^{-1} \mathbf{C}\left(\mathbf{C}^{H} \mathbf{R}^{-1} \mathbf{C}\right)^{-1} \mathbf{f} \\
\propto & \hat{\mathbf{a}}_{s}^{H} \mathbf{D} \mathbf{R}^{-1} \mathbf{D} \hat{\mathbf{a}}_{s} \times \mathbf{E}_{s} \mathbf{E}_{s}^{H} \mathbf{R}^{-1} \hat{\mathbf{a}}_{s} \\
& -\hat{\mathbf{a}}_{s}^{H} \mathbf{D} \mathbf{R}^{-1} \hat{\mathbf{a}}_{s} \times \mathbf{E}_{s} \mathbf{E}_{s}^{H} \mathbf{R}^{-1} \mathbf{D} \hat{\mathbf{a}}_{s} \\
\propto & \mathbf{E}_{s} \mathbf{E}_{s}^{H} \mathbf{R}^{-1}\left[\mathbf{I}-\frac{\hat{\mathbf{a}}_{s}^{H} \mathbf{D} \mathbf{R}^{-1} \hat{\mathbf{a}}_{s}}{\hat{\mathbf{a}}_{s}^{H} \mathbf{D} \mathbf{R}^{-1} \mathbf{D} \hat{\mathbf{a}}_{s}} \mathbf{D}\right] \hat{\mathbf{a}}_{s},
\end{aligned}
$$

where $\mathbf{f}=[1,0]^{T}, \hat{\mathbf{a}}_{s}=\mathbf{a}\left(\hat{\theta}_{s}\right)$, and $\mathbf{C}=\left[\hat{\mathbf{a}}_{s}, \mathbf{D} \hat{\mathbf{a}}_{s}\right]$ with $\mathbf{D}=\operatorname{diag}\left\{[0,1, \cdots, M-1]^{T}\right\}$. With the uses of $\hat{\theta}_{s}=\theta_{1}$ and the Taylor's series expansion [10], we have $\hat{\mathbf{a}}_{s}=\mathbf{a}_{1}+j \varepsilon \mathbf{D} \mathbf{a}_{1}$, where $\varepsilon=\pi\left[\sin \left(\theta_{1}\right)-\sin \left(\hat{\theta}_{s}\right)\right]$, such that the weight vector in (16) reduces to

$$
\begin{aligned}
\varepsilon & =\pi \mathbf{w} \approx \mathbf{E}_{s} \mathbf{E}_{s}^{H} \mathbf{R}^{-1}(\mathbf{I}-j \varepsilon \mathbf{D})(\mathbf{I}+j \varepsilon \mathbf{D}) \mathbf{a}_{1} \\
& =\mathbf{E}_{s} \mathbf{E}_{s}^{H} \mathbf{R}^{-1} \mathbf{a}_{1}+\varepsilon^{2} \mathbf{E}_{s} \mathbf{E}_{s}^{H} \mathbf{R}^{-1} \mathbf{D}^{2} \mathbf{a}_{1} \\
& =\mathbf{w}_{o}+\varepsilon^{2} \boldsymbol{\Gamma} \mathbf{b},\left[\sin \left(\theta_{1}\right)-\sin \left(\hat{\theta}_{s}\right)\right]
\end{aligned}
$$

where $\boldsymbol{\Gamma}=\mathbf{E}_{s} \mathbf{E}_{s}^{H} \mathbf{R}^{-1}, \mathbf{b}=\mathbf{D}^{2} \mathbf{a}_{1}$, and $\mathbf{w}_{o}=\mathbf{E}_{s} \mathbf{E}_{s}^{H} \mathbf{R}^{-1} \mathbf{a}_{1}$ denotes the optimal weight vector for the eigenspace beamformer without pointing error. The second term in
(17) is due to the estimation error in the first stage, which is insignificant when compared with $\mathbf{w}_{o}$ because of the fact that $\varepsilon \ll 1$.

\subsection{Algorithm Summary for the Proposed Two-Stage Beamformer}

The overall procedure of the proposed robust beamformer can be summarized as below.

(1) Obtain the sample averaged version of the received data correlation matrix $\hat{\mathbf{R}}$ given by

$$
\hat{\mathbf{R}}=\sum_{n=1}^{N_{s}} \mathbf{x}(n) \mathbf{x}^{H}(n) / N_{s},
$$

where $N_{s}$ denotes the number of the data samples.

(2) Obtain the preliminary DOA estimate $\theta_{s}$ for the desired source [11].

(3) Compute the refined DOA estimate $\hat{\theta}_{s}$ according to (15) with $\mathbf{R}$ replaced with $\hat{\mathbf{R}}$ in (18).

(4) Compute the weight vector $\mathbf{w}$ according to (16).

\subsection{Theoretical Analysis on the Output SINR}

In this subsection, the analysis expression of the output SINR associated with the proposed beamformer is presented. For a manageable analysis, we only consider a two-source system again. Substituting (6) and (17) into (11), along with some algebraic manipulations, the expansion of the output SINR for the proposed beamformer is approximately expressed by

$$
\begin{aligned}
\operatorname{SINR}_{o} & \approx \frac{\mathbf{a}_{1}^{H} \boldsymbol{\Gamma} \mathbf{a}_{1}}{1-\mathbf{a}_{1}^{H} \boldsymbol{\Gamma} \mathbf{a}_{1}}+\frac{\varepsilon^{4}\left(\mathbf{a}_{1}^{H} \boldsymbol{\Gamma} \mathbf{a}_{1} \mathbf{b}^{H} \boldsymbol{\Gamma} \mathbf{b}-\left|\mathbf{b}^{H} \boldsymbol{\Gamma} \mathbf{a}_{1}\right|^{2}\right)}{\mathbf{a}_{1}^{H} \boldsymbol{\Gamma} \mathbf{a}_{1}\left(1-\mathbf{a}_{1}^{H} \boldsymbol{\Gamma} \mathbf{a}_{1}\right)^{2}} \\
& =\operatorname{SINR}_{\text {opt }}+\frac{\varepsilon^{4}\left(\mathbf{a}_{1}^{H} \boldsymbol{\Gamma} \mathbf{a}_{1} \mathbf{b}^{H} \boldsymbol{\Gamma} \mathbf{b}-\left|\mathbf{b}^{H} \boldsymbol{\Gamma} \mathbf{a}_{1}\right|^{2}\right)}{\mathbf{a}_{1}^{H} \boldsymbol{\Gamma} \mathbf{a}_{1}\left(1-\mathbf{a}_{1}^{H} \boldsymbol{\Gamma} \mathbf{a}_{1}\right)^{2}}
\end{aligned}
$$

where

$$
\operatorname{SINR}_{\text {opt }}=\frac{\mathbf{a}_{1}^{H} \boldsymbol{\Gamma} \mathbf{a}_{1}}{1-\mathbf{a}_{1}^{H} \boldsymbol{\Gamma} \mathbf{a}_{1}}=\frac{\frac{\lambda_{1}^{2} \mathbf{e}_{1}^{H} \mathbf{e}_{1}}{\lambda_{1}+\sigma_{n}^{2}}+\frac{\lambda_{2}^{2} \mathbf{e}_{2}^{H} \mathbf{e}_{2}}{\lambda_{2}+\sigma_{n}^{2}}}{1-\frac{\lambda_{1}^{2} \mathbf{e}_{1}^{H} \mathbf{e}_{1}}{\lambda_{1}+\sigma_{n}^{2}}-\frac{\lambda_{2}^{2} \mathbf{e}_{2}^{H} \mathbf{e}_{2}}{\lambda_{2}+\sigma_{n}^{2}}} .
$$

Note that SINR ${ }_{\text {opt }}$ in (20) denotes the maximum output SINR obtained by the optimal beamformer (without pointing error). The result in (19) indicates that the proposed beamformer performs like the optimal LCMV beamformer with a slight degradation in output SINR, which is proportional to $\varepsilon^{4} \ll 1$.

\section{Computer Simulations}

Computer simulations were conducted to ascertain the performance of the proposed two-stage beamformer. The 
array employed was a sixteen-element $(M=16)$ uniformly linear array. All elements were assumed to be identical and omnidirectional. The scenario involved a desired source at $\theta_{1}=0^{\circ}$ with power $\sigma_{1}^{2}=1$, and $K-1$ uncorrelated interferers uniformly distributed over the angle range $\left(30^{\circ}, 50^{\circ}\right)$ with a varied signal power $\sigma_{2}^{2}$. The input SNR and SIR were defined as $\mathrm{SNR}_{i}=-10 \log _{10} \sigma_{n}^{2}$ and $\operatorname{SIR}_{i}=-10 \log _{10} \sigma_{2}^{2}$, respectively. Unless otherwise mentioned, the set of standard parameters

$$
\begin{aligned}
\mathrm{SNR}_{i} & =20 \mathrm{~dB} ; \quad \mathrm{SIR}_{i}=0 \mathrm{~dB} ; \\
K & =2 ; \quad \theta_{s}=5.3^{\circ} ; \quad N_{s}=\infty
\end{aligned}
$$

will be used throughout the section. It is noteworthy that $\theta_{s}=5.3^{\circ}$, which led to an error in DOA estimate of $0.3^{\circ}$ at the first stage, was chosen to investigate robustness against estimate error. Furthermore, the angle region of interesting was $B=20^{\circ} \quad\left(\theta_{s} \pm 10^{\circ}\right)$ and $N=10$ was used to determine the refined DOA estimate, which led to a DOA estimation error of $\pm 0.5^{\circ}$. For comparison, we also included the results obtained with the eigenspace [7], eigenspace with a first-order SDC (denoted by SDCeigenspace), and optimal beamformers, in which the optimal one utilized the correct look direction $\theta_{s}=\theta_{1}$ to compute the weight vector. Furthermore, the analysis results in (13) and (19) for the eigenspace and proposed beamformers, respectively, were also shown to ascertain their correctness.

The first set of simulations examines the output SINR of the proposed two-stage beamformer against white noise (input SNR). The corresponding results were shown in Figure 1. It is found that the output SINR values of the proposed scheme are close to those of the optimal one, confirming that the desired signal can be successfully retained and the interference can be effectively suppressed even in case of a large pointing error. Under a proper condition $\left(\mathrm{SNR}_{i}<5 \mathrm{~dB}\right.$ ), the eigenspace beamformer achieved a comparable performance as the optimal

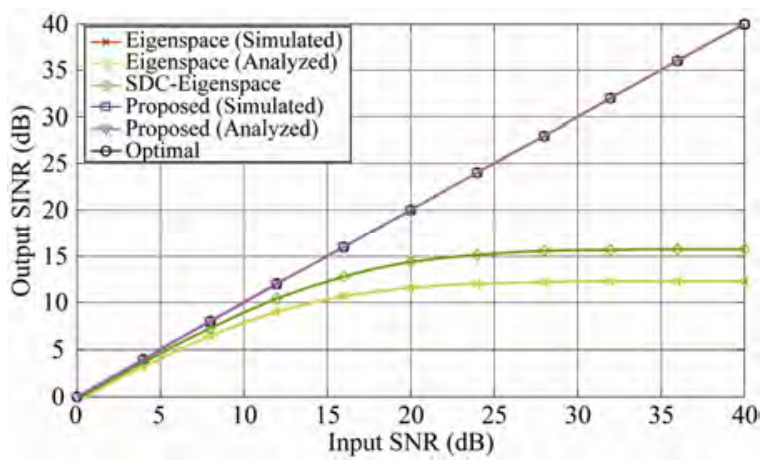

Figure 1. Output SINR performance versus input SNR with $M=16, \mathbf{S I R}_{\boldsymbol{i}}=\mathbf{0 ~ d B}, \theta_{s}=5.3^{\circ}, K=2$, and $N_{s}=\infty$. one. Unfortunately, for high input SNR (> $20 \mathrm{~dB}$ ), both the eigenspace-based beamformers, as expected, produced a significant degradation in output SINR. Furthermore, these beamformers reached the "saturation region" when the input SNR was larger than $20 \mathrm{~dB}$. This is because that the residual interference buried in the beamformer output cannot be negligible when compared with the output noise power, leading to a limitation in performance. It is noteworthy that the analyzed output SINR close to the simulated results confirms correctness of the theoretical analysis.

The second set of simulations investigates the effect of input SIR. Figure 2 shows the output SINR versus input SIR. It is observed that the proposed beamformer possessed an excellent robustness by effectively cancelling weak interference. On the contrary, the eigenspace beamformer failed to offer a reliable performance, especially for low input SIR $(<-10 \mathrm{~dB})$. Again, the reason for the significant discrepancy is that the pointing error effect induces a correlation between signals and makes the beamformer put less emphasis on suppressing interference. The analysis results approaching performance of the proposed beamformer confirm that the analysis results are correct.

The third set of simulations evaluates the effect of pointing errors on the proposed beamformer. In this case, the look direction $\theta_{s}$ was varied from $-10^{\circ}$ to $10^{\circ}$, corresponding to a maximum pointing error of $10^{\circ}$ (the null-to-null beamwidth of the broadside array is approximately $14.4^{\circ}$ ). Figure 3 shows the curves of output SINR versus $\theta_{s}$. The results indicate that desired signal cancellation does not occur even with the desired source located out of the "main-beam". On the other hand, both the conventional beamformers exhibit a significant degradation in SINR performance. Again, the correctness of the theoretical analysis was ascertained by achieving the similar results as the simulation results.

The fourth set of simulations examines the capability

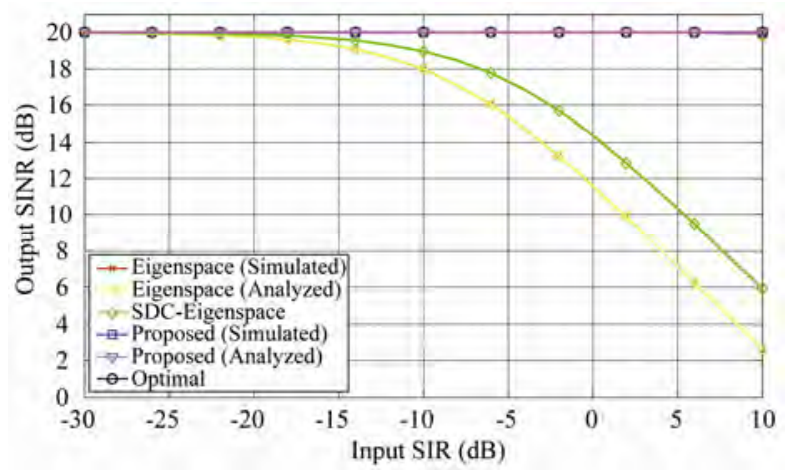

Figure 2. Output SINR performance versus input SIR with $M=16, \mathbf{S N R}_{\boldsymbol{i}}=\mathbf{2 0} \mathrm{dB}, \theta_{s}=5.3^{\circ}, K=2$, and $N_{s}=\infty$. 


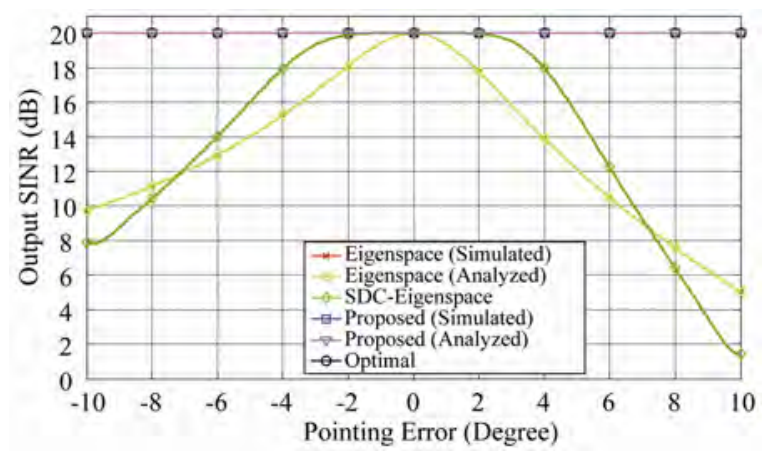

Figure 3. Output SINR performance versus pointing error with $M=16, \mathbf{S N R}_{\boldsymbol{i}}=\mathbf{2 0} \mathrm{dB}, \mathbf{S I R}_{\boldsymbol{i}}=\mathbf{0} \mathrm{dB}, K=2$, and $N_{s}=\infty$.

in interference suppression by varying the number of signal sources $K$. The resulting SINR plotted in Figure 4 indicates that desired signal cancellation did not occur with pointing error (no performance degradation) for $K<5$. In an interference-rich environment (large values of $K$ ), the non-zero cross correlation between signals makes the proposed scheme exhibit a certain degradation in performance due to the beam squint effect. The conventional eigenspace-based beamformers are sensitive to the number of interferers. These are confirmed by the beam patterns shown in Figure 5(a) and Figure 5(b) obtained with $K=5$ and 10, respectively. Clearly, all the beamformers successfully suppress interference even with a large pointing error. In the case of $K=5$, the proposed beamformer can resteer the beam back to the desired source direction to compensate for the error in the DOA estimate at the first stage. This did not happen with $K=10$ (an interference-rich environment). In addition, the conventional beamformers put a null in the direction of the desired signal, leading to a failure in beamforming.

The final set of simulations investigates the convergence behavior by varying the data sample size $N_{s}$ for computing the time-averaged version of the received data correlation matrix in (18). The results given in Figure 6 demonstrate that the proposed beamformer with a similar performance as the optimal one converges in about $N_{s}=10^{3}$ data samples, which is only about $0.37 \mathrm{~dB}$ away from the optimal case $\left(N_{s}=\infty\right)$. On the contrary, the other beamformers cannot collect the desired signal and completely suppress the interference even in the case of 5000 data samples due to the pointing error. To gain further insights, we show in Figure 7 the beam patterns obtained with $N_{s}=10^{3}$. We note that although the interferer was not perfectly cancelled, the proposed beamformer was still able to impose sufficient attenuation on it to prevent performance breakdown. On the other hand, the conventional beamformers cannot eliminate the interferer due to both the effects of pointing error and finite sample.

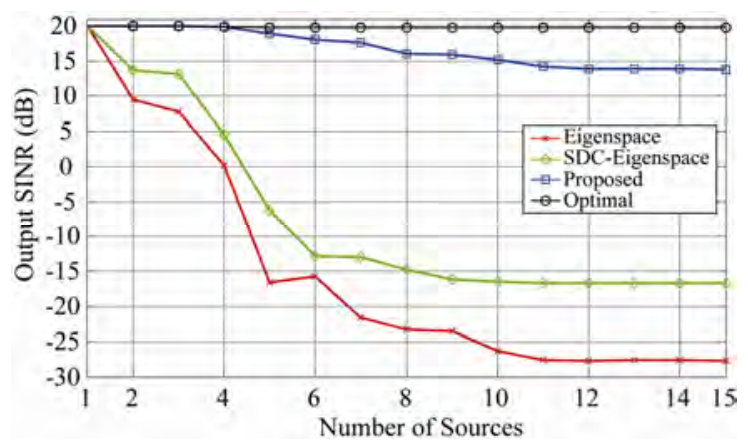

Figure 4. Output SINR performance versus $K$ with $M=16$, $\mathrm{SNR}_{\boldsymbol{i}}=20 \mathrm{~dB}, \mathrm{SIR}_{\boldsymbol{i}}=\mathbf{0} \mathbf{d B}, \theta_{\mathrm{s}}=5.3^{\circ}$, and $N_{\mathrm{s}}=\infty$.

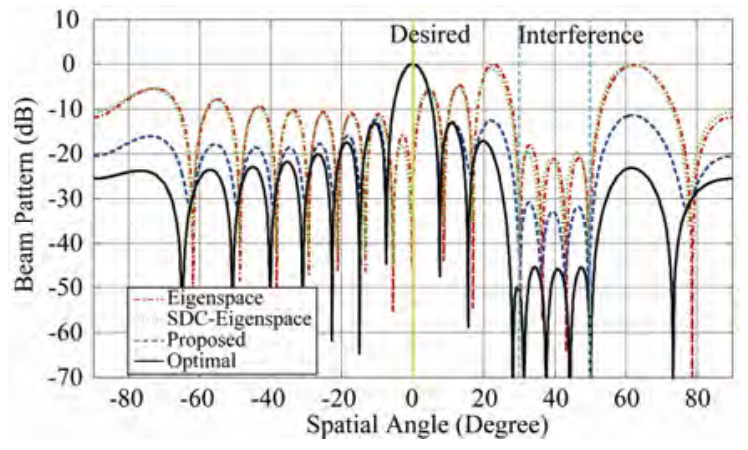

(a) $\mathrm{k}=5$

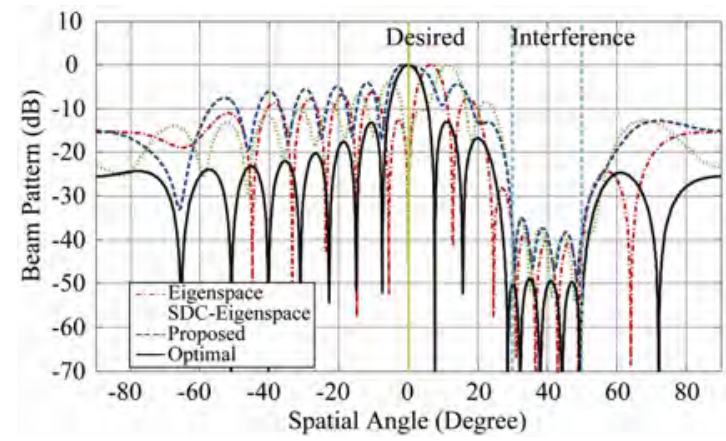

(b) $\mathrm{k}=10$

Figure 5. Beam pattern obtained with $M=16, \mathbf{S N R}_{\boldsymbol{i}}=20 \mathrm{~dB}$, $\mathbf{S I R}_{\boldsymbol{i}}=\mathbf{0 ~ d B}, \theta_{s}=5.3^{\circ}$, and $N_{s}=\infty$. (a) $K=5$; (b) $K=10$.

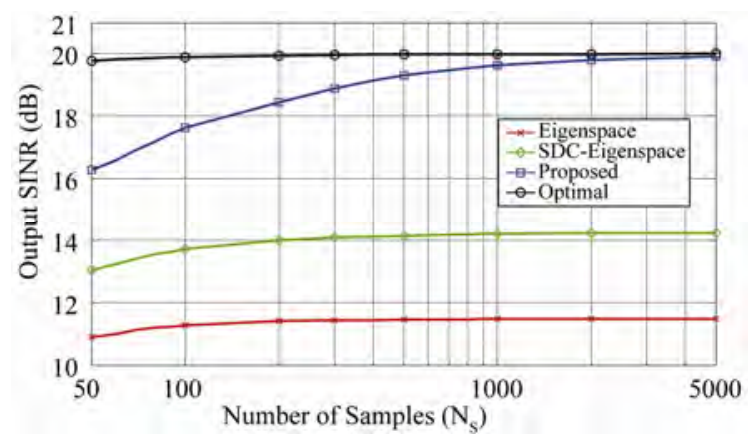

Figure 6. Output SINR performance versus sample size $N_{s}$ with $M=16, \mathbf{S N R}_{\boldsymbol{i}}=\mathbf{2 0} \mathbf{d B}, \mathbf{S I R}_{\boldsymbol{i}}=\mathbf{0 ~ d B}, K=2$, and $\theta_{s}=5.3^{\circ}$. 


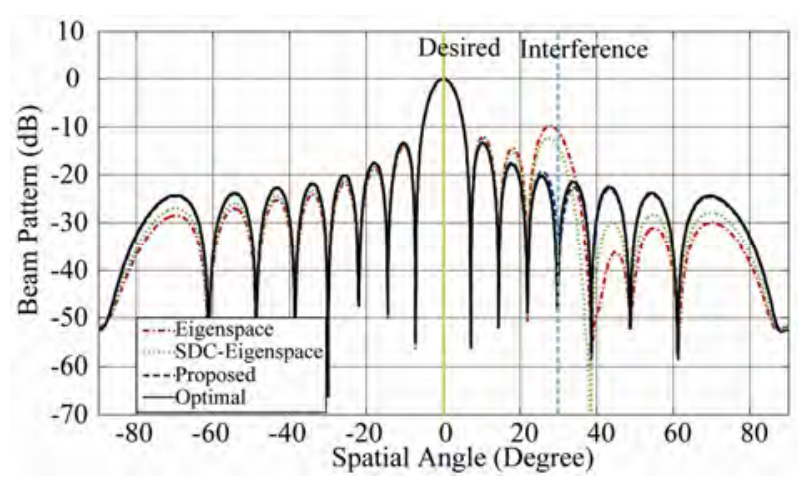

Figure 7. Beam pattern obtained with $M=16, \mathbf{S N R}_{\boldsymbol{i}}=\mathbf{2 0}$ $\mathbf{d B}, \mathbf{S I R}_{\boldsymbol{i}}=\mathbf{0 ~ d B}, K=2, \theta_{s}=5.3^{\circ}$, and $N_{s}=10^{3}$.

\section{Conclusions}

In this paper, we have derived an output SINR closedform expression of the eigenspace beamformer in terms of three important parameters including pointing errors, input SNR, and SIR. According to these analytical results, we find some intrinsic constraints imposed on the eigenspace beamformer. These constraints inspire us to develop a new beamforming scheme for combating large pointing errors. Computer simulations are presented to verify the derivation of the corresponding analysis. It is shown that the proposed beamformer possesses a better resistance to the pointing errors and excellent capability of suppressing weak interference in comparison with the conventional techniques, especially at a low input SIR.

\section{Acknowledgement}

This work was sponsored by the National Science Council, R. O. C, under the Contract NSC 99-2221-E-239023.

\section{REFERENCES}

[1] R. A. Monzingo and T. W. Miller, Introduction to Adaptive Arrays, New York, NY: John Wiley \& Sons, 1980.

[2] B. Widrow, K. M. Duvall, R. P. Gooch and W. C. Newman, "Signal Cancellation Phenomena in Adaptive Antennas: Causes and Cures," IEEE Trans. Antennas and Propagat., Vol. 30, No. 5, pp. 469-478, May 1982.

[3] B. D. Van Veen and K. M. Buckly, "Beamforming: A Versatile Approach to Spatial Filtering," IEEE ASSP Magazine, pp. 4-24, April 1988.

[4] M. H. Er, "Adaptive Antenna Array under Directional and Spatial Derivative Constraints," Proc. IEE, pt. H, Vol. 135, No. 6, pp. 414-419, 1998.

[5] K. C. Huang and C. C. Yeh, "Performance Analysis of Derivative Constraint Adaptive Arrays with Pointing Errors," IEEE Trans. Antennas and Propagat., Vol. 40, No. 8, pp. 975-981, Aug. 1992.

[6] Y. Chu and W. Y. Horng, "A Robust Algorithm for Adaptive Interference Cancellation," IEEE Trans. Antennas and Propagat., Vol. 56, No. 7, pp. 2121-2124, July 2008.

[7] L. Chang and C. C. Yeh, "Effect of Pointing Errors on the Performance of the Projection Beamformer,” IEEE Trans. Antennas and Propagat., Vol. 41, No. 8, pp. 1045-1056, Aug. 1993.

[8] M. H. Er and B. C. Ng, "A New Approach to Robust Beamforming in the Presence of Steering Vector Errors," IEEE Trans. Signal Process., Vol. 42, No. 7, pp. 18261829, July 1994.

[9] T. T. Lin, “A Two-Stage Beamformer with Enhanced Resistance Against Larger Pointing Errors," in Proc. PIMRC 2009, Tokyo, Japan, pp. 983-987, Spet. 2009.

[10] G. H. Golub and C. F. Van Loan, Matrix Computations, 2nd ed., Baltimore, MD: Johns Hopkins University Press, 1989.

[11] J. C. Liberti, T. S. Rappaport, Smart Antennas for Third Generation Mobile Communications, Artech House Publishers, 1998. 